domains (Lupus Symptoms, Physical Health, Pain-Vitality, Emotional Health and Body Image) were responsive to changes in patient reported and physician assessed health status (disease activity and damage) (Table 1). Procreation and Cognition domains showed responsive trends with patient reported change in health status, while Lupus Medications domain was responsive additionally to changes in Damage.

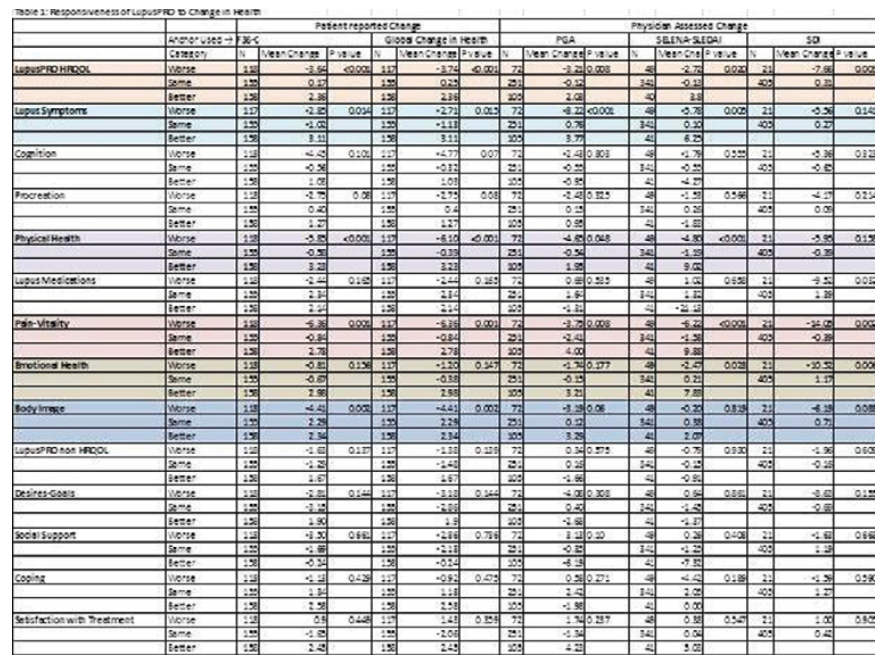

Conclusions: LupusPRO summary HRQOL and HRQOL domains show responsiveness to changes in patient-reported and physician assessed changes in health status in this observational study among Chinese SLE patients. Results support inclusion of LupusPRO into larger clinical trials to allow for robust estimates of responsiveness.

Disclosure of Interest: None declared

DOI: 10.1136/annrheumdis-2017-eular.3943

\section{THU0265 IDENTIFYING THE LINKS BETWEEN IRON DEFICIENCY AND FATIGUE IN ADOLESCENTS AND YOUNG ADULTS WITH SYSTEMIC LUPUS ERYTHEMATOSUS}

C. Wincup ${ }^{1}$, Y. loannou ${ }^{1}$, T. Richards ${ }^{2}$, F. Josephs ${ }^{1}$, L. Suffield ${ }^{1}$, A. Rahman ${ }^{1}$. ${ }^{1}$ Department of Rheumatology; ${ }^{2}$ Division of Surgery \& Interventional Science, University College London, London, United Kingdom

Background: Between $80-90 \%$ of patients with systemic lupus erythematosus (SLE) report fatigue to be the single most troublesome and debilitating symptom of their illness. ${ }^{1}$ Recent studies have found that functional iron deficiency and iron deficiency anaemia have been linked with fatigue and decreased cognitive performance. ${ }^{2}$ Increased red blood cell distribution width (RDW) is an early indicator of iron deficiency that can be useful in assessing iron stores in patients with SLE who may have an elevated serum ferritin due to underlying inflammation. Objectives: To investigate the relationship between early iron deficiency (measured by RDW) and fatigue in adolescents and young adults with SLE.

Methods: Adolescent and young adult patients with SLE were recruited prospectively between November 2016 and January 2017. All patients were asked to complete the Functional Assessment of Chronic Fatigue Illness Therapy (FACIT) Fatigue Scale $\mathrm{v} 4$, in which a numerical score between $0-52$ is generated. Lower scores indicate more fatigue. Standard measures of lupus disease activity including Erythrocyte Sedimentation Rate (ESR), C-Reactive Protein (CRP), Complement C3 levels, anti-double stranded DNA binding (anti-dsDNA) and SLEDAI were recorded. Haemoglobin $(\mathrm{Hb})$ and RDW were also measured. Anaemia was defined by World Health Organisation criteria (male $\mathrm{Hb}<130 \mathrm{~g} / \mathrm{L}$ and female $\mathrm{Hb}$ $<120 \mathrm{~g} / \mathrm{L})$. Non-parametric analysis was performed using Spearman's rank with a p-value $<0.05$ felt to be significant.

Results: 33 patients aged between 16.7 and 27.5 years (median age 20) were included. $85 \%$ of the patients were female. Their FACIT scores were lower than those published for healthy individuals of the same age group - median 24, IQR 22-44 for SLE vs median 43, IQR 35-48 for healthy. There was no statistically significant correlation between FACIT Fatigue score and SLEDAI $(p=0.92)$, antidsDNA ( $p=0.36)$, C 3 levels $(p=0.37)$, ESR $(p=0.30)$ or CRP $(p=0.85)$. Interestingly a statistically significant negative correlation between FACIT Fatigue score and RDW was observed $(p=0.012 ; r=-0.43)$. A correlation between FACIT Fatigue

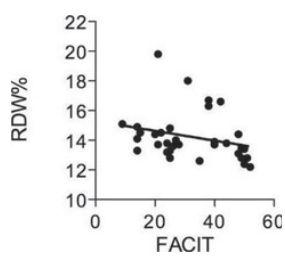

score and $\mathrm{Hb}$ was noted although this was not statistically significant $(\mathrm{p}=0.079)$. 12 of the 33 patients were found to be anaemic ( 11 female, 1 male). Analysis of the sub-group of 21 non-anaemic patients found FACIT Fatigue Score and RDW continue to show a statistically significant association $(p=0.026 ; r=-0.49)$.

Conclusions: Fatigue is a common and debilitating symptom described by young patients with SLE. Standard serological and clinical markers of disease activity did not correlate with the burden of fatigue. Increased RDW has been shown for the first time to correlate with increased fatigue in patients with lupus, suggesting that iron deficiency may play a significant role in the manifestation of this troublesome symptom. A trial of therapeutic iron infusions in the treatment of fatigue in SLE is planned.

References:

[1] Tench CM, McCurdie I, White PD, D'Cruz DP. The prevalence and associations of fatigue in systemic lupus erythematosus. Rheumatology 2000;39:12491254

[2] Greig AJ, Patterson AJ, Collins CE, Chalmers KA. Iron deficiency, cognition, mental health and fatigue in women of childbearing age: a systematic review. J Nutr Sci 2013:29:2:e14.

Disclosure of Interest: None declared

DOI: 10.1136/annrheumdis-2017-eular.2440

\section{THU0266 DAMAGE INDEXES IN PATIENTS WITH SYSTEMIC LUPUS ERYTHEMATOSUS AND SECONDARY ANTIPHOSPHOLIPID SYNDROME: DIAPS VS SLICC/ACR DAMAGE INDEX}

D. Potarniche ${ }^{1}$, D. Mazilu ${ }^{2}$, I. Saulescu ${ }^{2}$, A. Borangiu ${ }^{2}$, L. Groseanu ${ }^{2}$,

C. Constantinescu ${ }^{2}$, V. Vlad ${ }^{1}$, F. Berghea ${ }^{2}$, V. Bojinca ${ }^{2}$, D. Opris-Belinski ${ }^{2}$,

A. Balanescu" 2, D. Predeteanu 2, R. Ionescu" 2. "'Sfanta Maria" Clinical Hospital;

2"Sfanta Maria" Clinical Hospital, "Carol Davila" University of Medicine,

Bucharest, Romania, Bucharest, Romania

Background: Systemic lupus erythematosus (SLE) and antiphospholipid syndrome (APS) are systemic autoimmune diseases that have overlaping irreversible organ damages. Since SLICC/ACR Damage Index (SDI) misses key features of APS, the Damage Index in patients with Thrombotic Antiphospholipid Syndrome (DIAPS) was proposed.

Objectives: To assess the differences in indexes available for measuring organ damage in a cohort of patients with SLE and secondary APS.

Methods: Clinical records of patients with SLE and secondary APS were reviewed. Data on medical history and clinical manifestations were collected. The two damage indexes, SDI and DIAPS, were applied. Comparison between the two indexes was done for each organ system affected.

Results: Sixty five clinical charts were reviewed, 5 had been excluded for incomplete information. SDI and DIAPS was recorded in 60 patients. Patient's mean age was $45.05 \pm 14.61$ years, with mean disease duration of $9.47 \pm 6.96$ years. Mean SDI in our cohort was $4,15 \pm 2.58$ and mean DIAPS $-4.08 \pm 3.41$. SDI correlated significant to DIAPS $(R=0.826, p<0.000)$. Neuropsyhiatric manifestations were found in 25 patients $(41.7 \%)$. Their mean SDI value was $4.92 \pm 2.73$ and DIAPS value of $5.52 \pm 3.47$. DIAPS value was higher in the subgroup of patients with neuropsyhiatric $(\mathrm{p}=0.006)$ and respiratory system damage $(\mathrm{p}=0.037)$. This difference was not observed regarding SDI value. DIAPS value correlated significantly to neurological $(R=0.397, p=0.002)$ and pulmonary damage $(R=0.364, p=0.004)$, but not to SDI value. No diferrences were observed between the two scores regarding perypheral vascular manifestation (DIAPS $p=0.221$, $S D I p=0.136$ ) and renal involvement (DIAPS $p=0.062$, SDI $p=0.078$ ).

Conclusions: SDI may underestimate APS related damage in patients with SLE and secondary regarding neurological and pulmonary organ involvement. Given the implications for high morbidity and mortality, DIAPS may be the appropriate damage score to be used.

\section{References:}

[1] P. Alba et al. Organ Damage and Quality of Life in Antiphospholipid Syndrome. Current Rheumatology Reports. February 2016, 18:7.

[2] Barbhaiya et al. Utility of the Systemic Lupus International Collaborating Clinics (SLICC)/American College of Rheumatology (ACR) Damage Index for Antiphospholipid Antibody (aPL) Positive Patients. [abstract]. Arthritis Rheum 2011;63 Suppl 10:7.

Disclosure of Interest: None declared

DOI: 10.1136/annrheumdis-2017-eular.4384

\section{THU0267 METABOLIC SYNDROME AND HEALTH-RELATED QUALITY OF LIFE IN SYSTEMIC LUPUS ERYTHEMATOSUS}

D.P.E. Margiotta, F. Basta, M. Vadacca, G. Dolcini, F. Pignataro, A. Vernuccio, C. Mazzuca, L. Navarini, A. Afeltra. Unit of Allergology, Immunology and Rheumatology, Università Campus Bio-Medico di Roma, Rome, Italy, Rome, Italy

Background: Systemic Lupus Erythematosus (SLE) is associated to a huge prevalence and incidence of cardiovascular diseases (CVDs) due to accelerated atherosclerosis. Several evidences demonstrated that metabolic syndrome (MeS) could contribute to CVDs burden in SLE. In general population, MeS components and, according to some reports, MeS itself are associated to worsened Health related Quality of Life (HR-QoL). In SLE patients, a severe decline of HR-QoL has been widely demonstrated. 